A78 (1965).

${ }^{8}$ A. A. Lucas, J. Chem. Phys. $\underline{48}, 3156$ (1968).

${ }^{9}$ Benson and co-workers have found that oversimplified models which allow different displacements for different ions tend to overestimate ionic displacements significantly. See G. C. Benson and K. S. Yun, in The SolidGas Interface, edited by E. A. Flood (Marcel Dekker, New York, 1967), Vol. I, Chap. 8, and references therein.

${ }^{10}$ E. R. Cowley, J. Phys. C $\underline{4}, 988$ (1971).

${ }^{11}$ R. E. Allen, F. W. de Wette, and A. Rahman, Phys. Rev. 179, 887 (1969).

${ }^{12}$ If the rate of thermal expansion is larger at the surface than in the bulk, there will be a larger decrease in the surface mean-square amplitudes.

${ }^{13} \mathrm{As}$ a check on these results, an independent calculation was performed for the bulk, and good agreement was obtained with the results for the center of the slab. (See Table I. )

${ }^{14}$ A. Ignatjevs, T. N. Rhodin, S. Y. Tong, B. I. Lundqvist, and J. B. Pendry, Solid State Commun. 9,
1851 (1971).

${ }^{15}$ Compare Fig. 2 of Ref. 4 with Fig. 10 of R. E. Allen, G. P. Alldredge, and F. W. de Wette, Phys. Rev. B $\underline{4}, 1661$ (1971).

${ }^{16}$ The force-constant matrix is the matrix $K$ of Ref. 17 .

${ }^{17}$ R. M. Housley and F. Hess, Phys. Rev。 146, 517 (1966).

${ }^{18}$ See Eqs. (10) and (13) of Ref. 17 [with a typographical error to be corrected in Eq. (13), so that $m_{k}^{-1} \rightarrow m_{k}$ ].

${ }^{19}$ The high-temperature equality of the Debye-Waller factors for different ions in bulk models having only nearest-neighbor interactions has been conjectured by E. R. Cowley and R. A. Cowley, Proc. Roy. Soc. (London) A292, 209 (1966).

${ }^{20}$ See the discussion on p. 521 (right-hand column) of Ref. 17.

${ }^{21}$ J. S. Reid and T. Smith, J. Phys. Chem. Solids 31 , $2689(1970)$, and references therein.

${ }^{22}$ M. Merisalo and T. Paakkari, Acta Cryst. $\underline{23}, 1107$ (1967).

\title{
Surface Modes of Vibration in the Rigid-Ion Model of $\mathrm{NaCl}^{\dagger}$
}

\author{
T. S. Chen, G. P. Alldredge, and F. W. de Wette \\ Department of Physics, University of Texas, Austin, Texas 78712 \\ and \\ R. E. Allen \\ Department of Physics, Texas A \& $M$ University, College Station, Texas 77843 \\ (Received 15 February 1972)
}

\begin{abstract}
A discussion is given of the complete spectrum of vibrational surface modes for the Kellermann rigid-ion model of $\mathrm{NaCl}$. The attenuation with distance from the surface is shown for representative surface and pseudosurface modes. We discuss one interesting feature which has not previously been pointed out: Symmetry requirements can lead to surface modes which are associated with only one species of ion $\left(\mathrm{Na}^{+}\right.$or $\left.\mathrm{Cl}^{-}\right)$at the surface.
\end{abstract}

\section{INTRODUCTION}

There have been several discussions ${ }^{1-5}$ of surface modes in the Kellermann rigid-ion (KRI) model of $\mathrm{NaCl}$, but as yet no complete and accurate discussion has been given. Although for many purposes the results for the KRI model will be superceded by calculations based on the shell model ${ }^{4}$ and other improved models which are currently in progress, we feel that it is appropriate to give a complete discussion of the surface-mode spectrum for the KRI model before passing on to the shell model. The results for the KRI model yield considerable insight into the qualitative features that can be expected generally of surface and pseudosurface modes in ionic crystals.

\section{RESULTS}

The method for calculating the vibrational modes is discussed in detail elsewhere. ${ }^{2}$ Our procedure differs from that of Ref. 2 in only two respects, as discussed previously., 6 The surface ions relax inward, rather than outward, by a small amount, and a more rapidly convergent method is used to evaluate the Coulomb lattice sums.

In Fig. 1, the calculated frequencies of vibration $\omega_{p}(\bar{q})$ are shown for a 15-layer slab. Since there are two particles per unit cell and three directions of vibration, there are $2 \times 3 \times 15=90$ frequencies for each two-dimensional wave vector $\bar{q}$; i.e., $p=1,2, \ldots, 90$, where $p$ distinguishes the different normal modes which are associated with a given $\bar{q}$. The values of $\bar{q}$ for which the frequencies were determined lie along the symmetry lines of the two-dimensional Brillouin zone, $\bar{\Gamma} \overline{\mathrm{X}}, \overline{\mathrm{X}} \overline{\mathrm{M}}$, and $\overline{\mathrm{M}} \bar{\Gamma}$, as shown in Fig. 2(c). In Figs. 2(a) and (b) we show the frequencies for values of $\bar{q}$ along two lines $(\bar{\Gamma} \bar{Y})$ which make angles of $30^{\circ}$ and $15^{\circ}$, respectively, with the line $\bar{\Gamma} \bar{M}$. (The endpoints of 


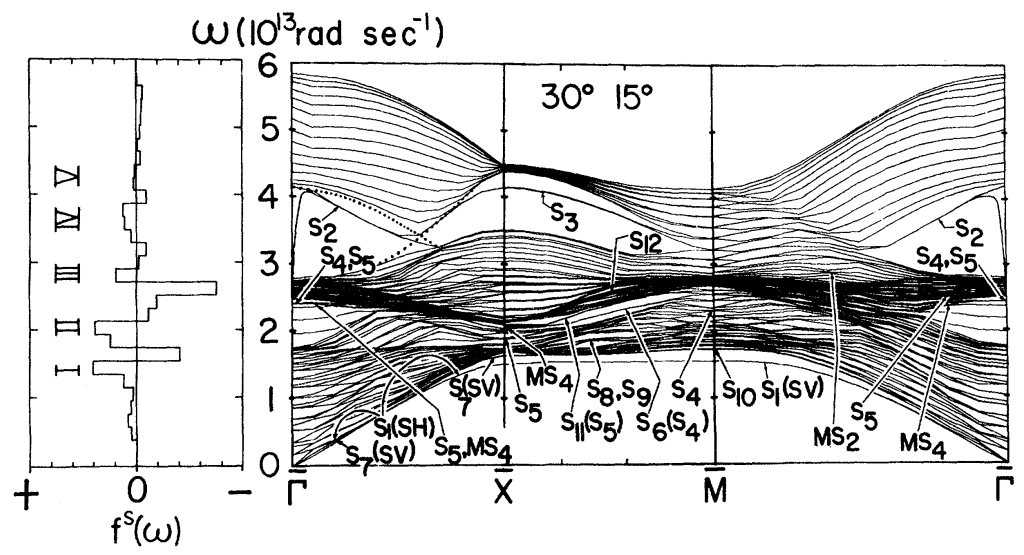

FIG. 1. Vibrational frequencies $\omega$ for 15-layer slab versus wavevector $\bar{q}$, for values of $\bar{q}$ along the symmetry lines shown in Fig. 2(c). The curves are computer generated, and crossovers between branches are not taken into account. Each pair of surface modes $S_{i}$, $i=1,2, \ldots, 12$, is labeled according to an arbitrary scheme. On the lefthand side, a histogram is given which approximates the surface frequency-distribution function $f^{s}(\omega)$. these lines, along $\overline{\mathrm{X}} \overline{\mathrm{M}}$, are indicated in Fig. 1.) On the left-hand side of Fig. 1, we show the surface frequency-distribution function $f^{s}(\omega)$ (horizontal axis) versus the frequency $\omega$ (vertical axis); $f^{s}(\omega)$ is defined by

$$
f^{s}(\omega)=(N / n)\left[f(\omega)-f^{b}(\omega)\right],
$$

where $N$ is the total number of ions in the slab, and $n$ is the number of surface ions. Here $f(\omega)$ is the frequency-distribution function for the slab, defined such that $f(\omega) d \omega$ is the fraction of the vibrational frequencies between $\omega$ and $(\omega+d \omega)$. A mesh of 66 sample $\bar{q}$ values were used throughout the irreducible element [the region surrounded by heavy lines in Fig. 2(c)] in determining $f(\omega)$; there were thus $66 \times 90=5940$ independent sample frequencies (for a total of 36000 frequencies in the whole zone). The bulk frequency-distribution function $f^{\circ}(\omega)$ was determined in an independent calculation involving 422 values of the wave vector in the irreducible element of the three-dimensional Brillouin zone, and thus $422 \times 2 \times 3=2532$ independent sample frequencies (a total of 82944 frequencies in the Brillouin zone). The regions where $f^{s}(\omega)$ is positive in our histogram are labeled by Roman numerals (I-V).

There are four pairs of surface- or pseudosurface-mode branches which exist at large wave- lengths along $\bar{\Gamma} \bar{M}$ : In chronological order of discovery, these are the Rayleigh ${ }^{7}$ modes $S_{1}$, the Fuchs-Kliewer ${ }^{8}$ (FK) modes $S_{2}$, and the Lucas ${ }^{1}$ modes $S_{4}$ and $S_{5}$. The Rayleigh modes are acoustical surface modes which are obtained in elasticity theory. The FK modes are optical surface modes which are obtained in the continuum theory of vibrations in ionic crystals. The Lucas modes are "microscopic" surface modes which penetrate only a few atomic spacings beneath the surface and can therefore be obtained only in a microscopic theory, i.e., lattice dynamics. The Lucas modes are "peeled off" from the "bands" of transverse-optical (TO) bulk modes by short-range changes in the force constants at the surface; similar modes which are "peeled off" from the longitudinal-optical (LO) bulk bands have been found in shell-model calculations for $\mathrm{RbF}^{4(\mathrm{a}, \mathrm{b})}$ and for $\mathrm{RbCl}, \mathrm{NaI}$, and $\mathrm{NaBr} .^{4(b)}$

As $\bar{\Gamma}$ is approached from any direction [left- and right-hand sides of Figs. 1, 2(a), and 2(b)], the FK modes separate in frequency because they penetrate more and more deeply. ${ }^{9}$ The upper branch enters the LO bulk band as a series of pseudosurface (or mixed) modes, ending at the top of the LO band at the point $\bar{\Gamma}$. The lower branch similarly enters the TO bulk band as a series of pseudosurface modes, ending at the bottom of the TO band at
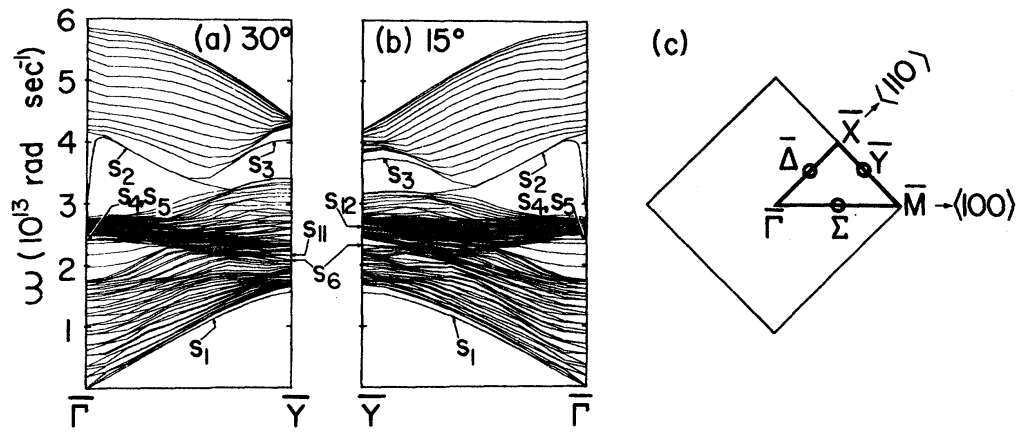

FIG. 2. (a) Frequencies $\omega$ versus wavevector $\bar{q}$ for values of $\bar{q}$ along a line making an angle of $30^{\circ}$ with the line $\bar{\Gamma} \bar{M}$. (b) $\omega$ versus $\bar{q}$ for a line $15^{\circ}$ from $\bar{\Gamma} \bar{M}$. (c) Two-dimensional Brillouin zone for the (100) surface of NaCl. The crystallographic directions $\langle\mathbf{1 1 0}\rangle$ and $\langle 100\rangle$ parallel to the surface are indicated. 


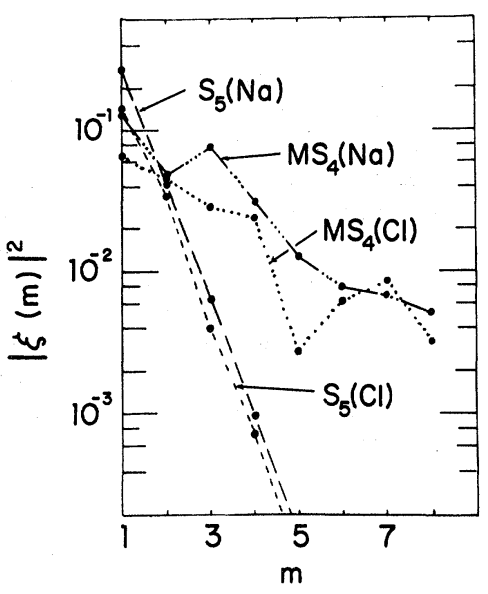

FIG. 3. $|\vec{\xi}(m \kappa)|^{2}$ versus $m$ for the surface mode $S_{5}$ and the mixed mode $M S_{4}$ at a point along $\bar{\Gamma} \bar{M}$ which is $\frac{1}{5}$ of the distance from $\bar{\Gamma}$ to $\overline{\mathrm{M}}$ and is indicated by the arrows in Fig. 1. Here and in Figs. 4-8, the value of $|\vec{\xi}|^{2}$ is the average for both members of the pair of nearly degenerate surface modes labeled by $S_{i}$.

$\bar{\Gamma}$. This behavior, which was first discussed by Jones and Fuchs, ${ }^{5}$ is not clear in Figs. 1 and 2 because the curves were computer generated for a finite mesh of $\bar{q}$ values ${ }^{10}$; however, it is shown schematically in Fig. 1 of Ref. 4.

As $\bar{q}$ increases from zero, the FK modes merge in Figs. 1 and 2 (i.e., their frequencies become nearly degenerate), and the two branches emerge into the gap between LO and TO bulk modes as a well-defined pair of surface modes $S_{2}$. As $\bar{q}$ continues to increase, however, this gap closes up as indicated by the dotted lines at the left-hand side of Fig. 1. (These lines represent the lower edge of the LO bulk band and the upper edge of the TO bulk bands, as determined in an independent calculation of the bulk modes.) The FK modes, therefore, enter the bulk bands and are no longer surface modes. The mixed mode $M S_{2}$ on the righthand side of Fig. 1 is, in effect, an extension of $S_{2}$ into the bulk bands.

The Lucas modes occur as two pairs of surface modes, $S_{4}$ and $S_{5}$, which are degenerate because of symmetry at $\bar{\Gamma}$. As $\bar{q}$ increases from zero along a general direction [e.g., Figs. 2(a) and 2(b)], each pair of Lucas modes becomes a series of mixed modes, $M S_{4}$ and $M S_{5}$, because they couple to the modes of the surrounding bulk bands. However, along $\bar{\Gamma} \overline{\mathrm{X}}$ and $\bar{\Gamma} \overline{\mathrm{M}} S_{5}$ is symmetry decoupled from the surrounding bulk modes, ${ }^{11}$ and so it continues to exist as a surface mode along these symmetry lines. Also, $S_{4}$ is symmetry decoupled from the bulk modes surrounding it at $\bar{M},{ }^{12}$ so it is again a surface mode at this point.

In Figs. 3 and 4 we show the attenuation of the Lucas modes (as surface modes and mixed modes) with distance from the surface. Here $\xi_{\alpha}(m \kappa)$ is the component of the eigenvector ${ }^{13}$ associated with a given vibrational mode for the $\alpha$ direction $(\alpha=x$, $y$, or $z$ ), the $m$ th plane of ions (with $m=1$ at the surface), and the $\kappa$ ion $\left(\kappa=\mathrm{Na}^{+}\right.$or $\left.\mathrm{Cl}^{-}\right)$. The vibrational amplitude of the ion, $u_{\alpha}(m \kappa)$, is related to the eigenvector in the following way ${ }^{14}$ :

$$
\left|u_{\alpha}(m \kappa)\right|^{2} \propto M_{\kappa}^{-1}\left|\xi_{\alpha}(m \kappa)\right|^{2} .
$$

We have plotted, in Figs. 3-8,

$$
|\vec{\xi}|^{2}=\left|\xi_{x}\right|^{2}+\left|\xi_{y}\right|^{2}+\left|\xi_{z}\right|^{2}=M_{k}|\overrightarrow{\mathrm{u}}|^{2}
$$

versus the layer index $m$ for both the $\mathrm{Na}^{+}$and $\mathrm{Cl}^{-}$ ions. In Fig. 3, for a value of $\bar{q}$ near $\bar{\Gamma}$ along $\bar{\Gamma} \overline{\mathrm{M}}$, it is apparent that $S_{5}$ is indeed a surface mode, since its amplitude goes rapidly (and approximately exponentially) to zero with increasing distance from the surface. It is also fairly clear that $M S_{4}$ is (as it must be) a mixed mode whose amplitude would not approach zero (in a semiinfinite crystal) with increasing distance from the surface. The same conclusions hold for $S_{5}$ and $M S_{4}$ at the point $\overline{\mathrm{X}}$ in Fig. 4. In Fig. 5, it is clear that $S_{4}$ is indeed a surface mode at the point $\bar{M}$.

In Fig. 5, the attenuation is also shown for the surface mode $S_{10}$, which lies under the acoustical bulk bands near the point $\overline{\mathrm{M}} . \quad S_{10}$ and $S_{1}$ are related in a rather interesting way: Both have predominantly $S V$ polarization (i.e., they are polarized mainly in the direction normal to the surface). Near $\bar{\Gamma}, S_{1}$ penetrates deeply and is associated with vibrations in all the planes near the surface for both $\mathrm{Na}^{+}$and $\mathrm{Cl}^{-}$ions. As $\overline{\mathrm{M}}$ is approached along $\bar{\Gamma} \bar{M}$, however, $S_{1}$ becomes associated primarily with vibrations in the first layer for $\mathrm{Cl}^{-}$ions and vibrations in the second layer for $\mathrm{Na}^{+}$ions. At $\overline{\mathrm{M}}$, in fact, $S_{1}$ has zero amplitude in the second,

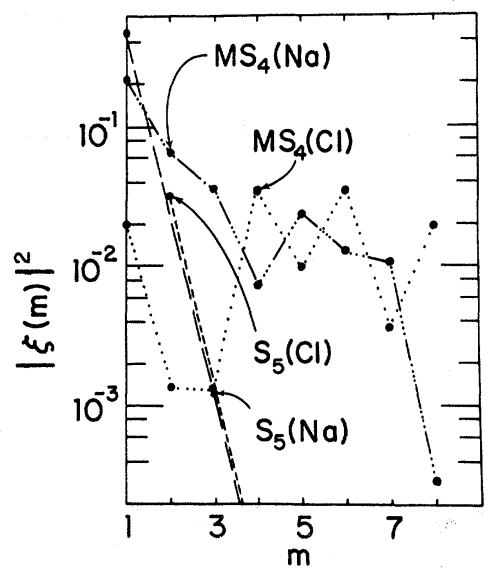

FIG. 4. $|\vec{\xi}(m \kappa)|^{2}$ versus $m$ for $S_{5}$ and $M S_{4}$ at the point $\overline{\mathrm{X}}$. In this and the following figures, points for which $|\vec{\xi}|^{2}$ $=0$ are not plotted, and the points for which $|\vec{\xi}|^{2} \neq 0$ are connected by lines. 


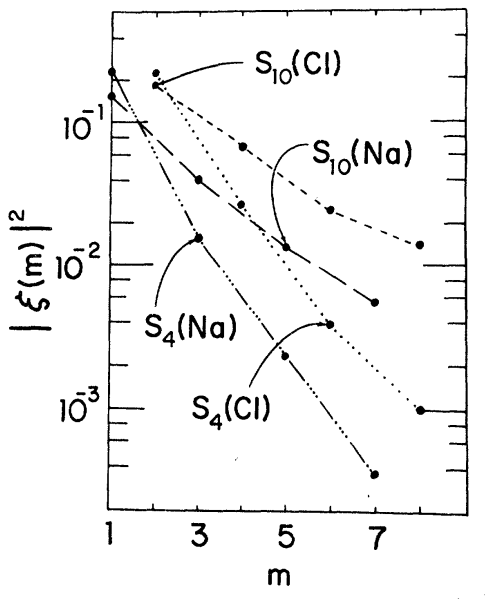

FIG. 5. $|\vec{\xi}(m \kappa)|^{2}$ versus $m$ for $S_{4}$ and $S_{10}$ at the point $\bar{M}$.

fourth, etc., layers for $\mathrm{Cl}^{-}$ions and zero amplitude in the first, third, etc., layers for $\mathrm{Na}^{+}$ions, as can be seen in Fig. 6 . On the other hand, $S_{10}$ has zero amplitude in the odd layers for $\mathrm{Cl}^{-}$ions and in the even layers for $\mathrm{Na}^{+}$ions, as can be seen in Fig. 5. Both $S_{1}$ and $S_{10}$ exhibit a roughly exponential decrease in amplitude with distance from the surface for the layers where the amplitude is nonzero. At $\overline{\mathrm{M}}$, both $S_{1}$ and $S_{10}$ have strictly $S V$ polarization.

The behavior of $S_{1}$ and $S_{10}$ described above can be understood in the following way: Because of the symmetry at $\bar{M}$, one can show that at this point there is a decoupling of the $\mathrm{Na}^{+}$ions in odd layers and $\mathrm{Cl}^{-}$ions in even layers from the $\mathrm{Na}^{+}$ions in even layers and $\mathrm{Cl}^{-}$ions in odd layers for vibrations normal to the surface. ${ }^{15}$ Consequently, the $S V$-polarized modes at $\overline{\mathrm{M}}$ are associated either with vibrations of $\mathrm{Na}^{+}$ions in odd layers and $\mathrm{Cl}^{-}$ions in even layers or else with vibrations of $\mathrm{Na}^{+}$in even layers and $\mathrm{Cl}^{-}$in odd layers. It is therefore reasonable that we should have two $S V$ modes at $\overline{\mathrm{M}}$, one associated with $\mathrm{Cl}^{-}$surface ions and the other with $\mathrm{Na}^{+}$surface ions. Since the $\mathrm{Cl}^{-}$ion is heavier and the force constants for $\mathrm{Cl}^{-}$and $\mathrm{Na}^{+}$ions are equal in our model, the lower frequency surface mode $S_{1}$ is associated with $\mathrm{Cl}^{-}$surface ions.

Between $\overline{\mathrm{M}}$ and $\overline{\mathrm{X}}, S_{1}$ undergoes a change of polarization, until at $\overline{\mathrm{X}}$ it has strictly $S H$ polarization. Another surface mode $S_{7}$ appears near $\overline{\mathrm{X}}$ and acquires the $S V$ polarization lost by $S_{1}$. Along $\bar{\Gamma} \overline{\mathrm{X}}$, there are two acoustical surface modes which would appear to persist into the long wavelength regime for a semi-infinite medium $-S_{1}$, with $S H$ polarization, and $S_{7}$, which is polarized within the sagittal plane. Between $\bar{\Gamma}$ and $\overline{\mathrm{X}}, S_{1}$ and $S_{7}$ cross, as indicated in Fig. $1 .^{16}$ At long wavelengths, therefore, the lowest acoustical surface mode always has predominantly $S V$ polarization. This result is in agreement with our general expectations

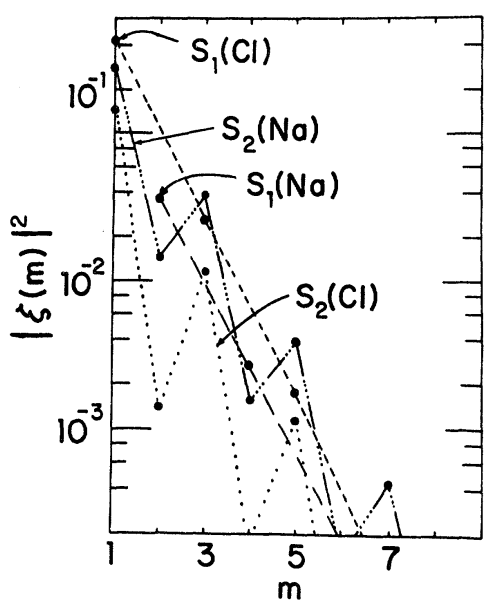

FIG. 6. $|\vec{\xi}(m \kappa)|^{2}$ versus $m$ for $S_{1}$ at the point $\bar{M}$ and for $S_{2}$ at the same point along $\bar{\Gamma} \bar{M}$ used in Fig. 3 and indicated by the arrow in Fig. 1 .

for materials having anisotropy ratio $\eta\left[=2 C_{44} /\right.$ $\left.\left(C_{11}-C_{12}\right)\right]$ less than unity, such as $\mathrm{NaCl} .^{17,18}$ As has been pointed out previously, ${ }^{17}$ it would be interesting to see if the $S H$ surface mode $\left(S_{1}\right)$ can be obtained along $\bar{\Gamma} \overline{\mathrm{X}}$ (i.e., the $\langle 110\rangle$ direction) in the longwavelength regime for $\mathrm{NaCl}$ and other material with $\eta<1$.

In addition to the surface modes discussed above, there are a number of others which occur near the Brillouin-zone boundary in gaps between the bulk bands-i.e., $S_{3}, S_{6}, S_{8}, S_{9}, S_{11}$, and $S_{12}$. It is probably not worthwhile to discuss these modes in detail, because the gaps within the bulk bands-and thus the nature of the surface modes within these gaps-change considerably in going over from the rigid-ion model to the shell model. The important point is that a variety of such "gap modes" do in fact occur. In Fig. 7, we show the attenuation of

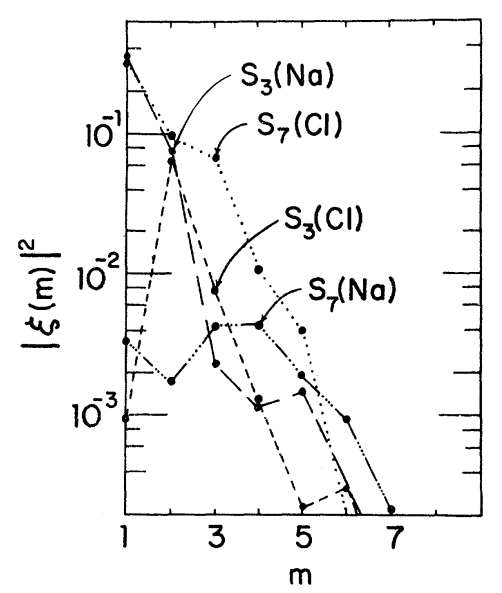

FIG. 7. $|\vec{\xi}(m \kappa)|^{2}$ versus $m$ for $S_{3}$ and $S_{7}$ at $\overline{\mathrm{X}}$. 


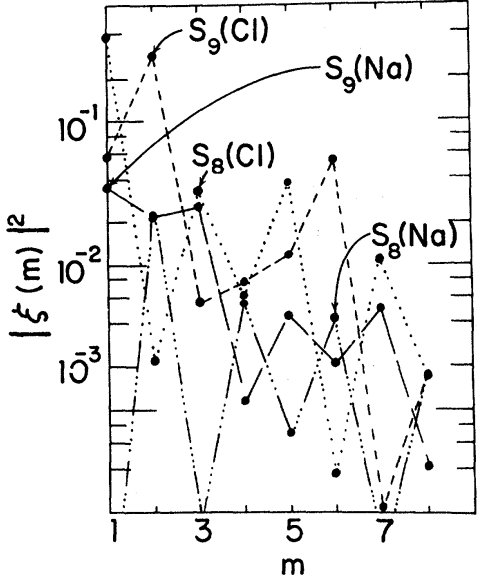

FIG. 8. $|\vec{\xi}(m \kappa)|^{2}$ versus $m$ for $S_{8}$ and $S_{9}$ at the point indicated by the arrow in Fig. 1.

the most prominent "gap mode," $S_{3}$. (This mode is also prominent in the results of shell-model calculations. ${ }^{4}$ ) In Fig. 8, the (rather complicated) attenuation of $S_{8}$ and $S_{9}$ is shown. Two of the "gap modes" are, in effect, extensions of the Lucas modes: $S_{6}$ corresponds to the Lucas mode $S_{4}$, and $S_{11}$ corresponds to $S_{5}$, as indicated in Fig. 1 .

\section{CONCLUSION}

Although the surface-mode spectrum of $\mathrm{NaCl}$ (100) as calculated with the rigid-ion model is not accurate quantitatively, the results for this model do provide qualitative insight into what can generally be expected of surface and pseudosurface modes in ionic crystals. Among the qualitative features of general interest are the following: (a) Both the acoustical (Rayleigh) and optical (Fuchs-Kliewer) surface modes obtained in continuum calculations are also obtained in lattice-dynamical calculations. (b) For zero wave vector, optical surface modes are obtained which are "peeled off" from bands of the bulk optical modes. In the present case, these (Lucas) modes are peeled off from the TO bands. (c) Numerous surface-mode branches are found within gaps in the bulk bands, particularly near the Brillouin-zone boundary. (d) Surface modes sometimes lie within the bulk bands along symmetry lines $\left(S_{5}\right.$ along $\bar{\Gamma} \overline{\mathrm{M}}$ and $\bar{\Gamma} \overline{\mathrm{X}}$ ) or at symmetry points $\left(S_{4}\right.$ at $\bar{\Gamma}$ and $\left.\bar{M}\right)$. (e) Sometimes surface modes are found which have large amplitude at the surface for one ion (e.g., $\mathrm{Cl}^{-}$) and small amplitude at the surface for the other ion (e.g., $\mathrm{Na}^{+}$). This is the case near the point $\bar{M}$, where symmetry requirements result in two surface modes with predominantly $S V$ polarization $-S_{1}$, which is associated with the vibrations of $\mathrm{Cl}^{-}$ions at the surface, and $S_{10}$, which is associated with vibrations of $\mathrm{Na}^{+}$ions at the surface. It is interesting that much of the behavior of surface modes, in both monatomic ${ }^{18}$ and nonmonatomic crystals, can be explained with symmetry arguments.

Finally, we mention that our results indicate the presence of two acoustical surface waves $\left(S_{1}\right.$ and $S_{7}$ ) which apparently persist into the long-wavelength regime along the $\langle 110\rangle$ direction. It would be interesting to see whether the $S H$ surface wave $S_{1}$ (and corresponding pseudosurface waves for directions close to $\langle 110\rangle)$ can be obtained in the long-wavelength regime for $\mathrm{NaCl}$ and other materials which have anisotropy ratios less than unity.
†Research sponsored in part by the U. S. Air Force Office of Scientific Research (A FSC) under Grant No. AFOSR 71-1973, and in part by the Robert A. Welch Foundation.

${ }^{1}$ A. A. Lucas, J. Chem. Phys. 48, 3156 (1968).

${ }^{2}$ S. Y. Tong and A. A. Maradudin, Phys. Rev. 181, 1318 (1969).

${ }^{3}$ T. S. Chen, R. E. Allen, G. P. Alldredge, and F. W. de Wette, Solid State Commun. 8 , 2105 (1970). The numerous printing errors in this paper are corrected in an erratum: 9 , No. 16 , xi (1971).

${ }^{4}$ (a) T. S. Chen, G. P. Alldredge, F. W. de Wette, and R. E. Allen, Phys. Rev. Letters 26, 1543 (1971); (b) T. S. Chen, G. P. Alldredge, and F. W. de Wette, Solid State Commun. (to be published).

${ }^{5}$ W. E. Jones and R. Fuchs, Phys. Rev. B $\underline{4}, 3581$ (1971).

${ }^{6}$ T. S. Chen, G. P. Alldredge, F. W. de Wette, and R. E. Allen, preceding paper, Phys. Rev. B $\underline{6}, 623$ (1972).

${ }^{7}$ Lord Rayleigh, London Math. Soc. Proc. 17, 4 (1885).

${ }^{8}$ R. Fuchs and K. L. Kliewer, Phys. Rev. 140 , A2076 (1965).

${ }^{9}$ Surface modes in a slab occur in pairs which are nearly degenerate unless they are deeply penetrating, as is the case for the Rayleigh and FK modes at long wavelengths.

${ }^{10}$ In Fig. 1 the curved line representing the lower branch of $S_{2}$ was drawn in, using results for values of $\bar{q}$ very close to $\bar{\Gamma}$. The rest of the lines are computer generated.

${ }^{11}$ Along $\bar{\Gamma} \overline{\mathrm{X}}$ and $\bar{\Gamma} \overline{\mathrm{M}}, S_{5}$ has strictly $S H$ polarization; i.e., it is polarized strictly perpendicular to the sagittal plane, which includes $\bar{q}$ and the normal to the surface. $M S_{4}$ and the surrounding acoustical bulk modes are polarized strictly within the sagittal plane.

${ }^{12} \mathrm{At} \overline{\mathrm{M}}, S_{4}$ is polarized strictly perpendicular to the surface, and the bulk modes surrounding it are polarized strictly in the other two directions.

${ }^{13}$ See Eq. (3) of Ref. 6. The planar indices $m$ and $l_{3}$ are related in that $m=1$ at the surface and $l_{3}=0$ at the center of the slab.

${ }^{14}$ R. E. Allen, G. P. Alldredge, and F. W. de Wette, Phys. Rev. B $\underline{4}, 1648$ (1971); see Eq. (2.15).

${ }^{15} \mathrm{~A}$ similar effect in monatomic fcc crystals with shortrange interactions leads to the presence of "single-layer modes" at $\overline{\mathrm{M}}$ which have $S V$ polarization. See p. 1668 of Ref. 18.

${ }^{16}$ The crossing of $S_{1}$ and $S_{7}$ along $\bar{\Gamma} \overline{\mathrm{X}}$ does not occur in the more reliable shell-model calculations; see Ref. 4. (In these latter calculations for $\mathrm{NaCl}, S_{1}$ retains $S V$-like 
polarization between $\overline{\mathrm{M}}$ and $\overline{\mathrm{X}}$, and $S_{7}$ is $S H$-like and is well above $S_{1}$ all along $\bar{\Gamma} \overline{\mathrm{X}}$.)

${ }^{17}$ G. P. Alldredge, R. E. Allen, and F. W. de Wette,
J. Acoust. Soc. Am. $\underline{49}, 1453$ (1971).

${ }^{18}$ R. E. Allen, G. P. Alldredge, and F. W. de Wette, Phys. Rev. B $\underline{4}, 1661$ (1971).

\title{
Surface Vibrations in a Model hcp Crystal*
}

\author{
R. E. Allen \\ Department of Physics, Texas A \& $M$ University, College Station, Texas 77843 \\ and \\ G. P. Alldredge and F. W. de Wette \\ Department of Physics, University of Texas, Austin, Texas 78712 \\ (Received 16 February 1972)
}

\begin{abstract}
The surface relaxation, vibrational modes, mean-square amplitudes of vibration, etc., have been calculated for the (0001) surface of a model hcp crystal in which the particles interact through a Lennard-Jones potential. The results are virtually indistinguishable from those for the (111) surface of a fcc crystal.
\end{abstract}

In a previous paper, ${ }^{1}$ we reported a study of the surface-mode spectrum for the (111) surface of a model fcc crystal in which the particles are taken to interact through a Lennard-Jones potential. Here we report a study of the surface vibrations in a model crystal with the same interaction potential which has an hcp structure and a (0001) surface. The two models differ only in that the former has an $A B C A B C .$. stacking and the latter has an $A B A B$... stacking.

We have followed the same procedure as described for the fcc crystals in our earlier papers $^{1,2}$ : First the static relaxation at the surface is determined by minimizing the static energy, then the vibrational frequencies and associated eigenvectors are determined, and finally physical quantities such as the mean-square amplitudes are calculated.

We find that the results of our calculations-for the relaxation, vibrational frequencies, meansquare amplitudes of vibration, etc., -are virtually indistinguishable from those obtained previously for the (111) surface of a fcc crystal. As one example, we show in Fig. 1 the results for the vibrational frequencies, which are almost exactly the same as those shown in Fig. 8 of Ref. 1. As another example, we mention that, contrary to an expectation that the short-wavelength surface modes might reflect the difference in stacking sequence
( $A B A B$ versus $A B C A B C$ ), the depth dependence of the surface modes $S_{1}, S_{2}, S_{3}$, and $S_{4}$ (when plotted for comparison with Fig. 9 of Ref. 1) displays no significant effect of the different stacking sequences.

On the basis of these results, we expect the same general behavior for surface vibrations at the (111) surfaces of monatomic fcc materials and (0001) surfaces of hcp materials.

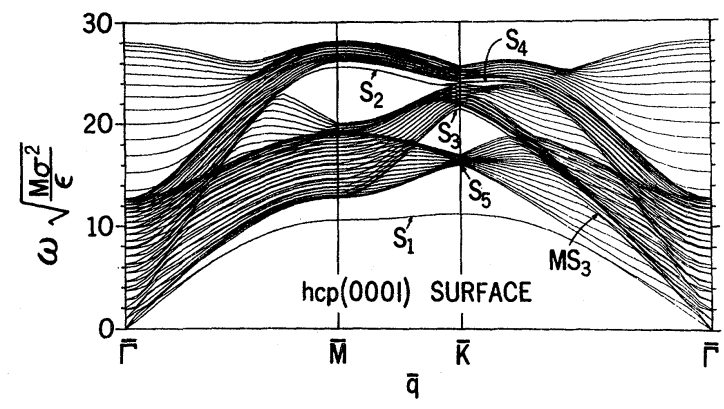

FIG. 1. Vibrational frequencies versus planar wave vector q for 21-layer slab with hcp structure and (0001) surfaces, determined in the same manner as in Ref. 1 with the surface relaxation taken into account. These results are virtually indistinguishable from those in Fig. 8 of Ref. 1 for a fcc structure and (111) surfaces. $(\bar{\Gamma}$ is the origin, and $\bar{\Gamma} \bar{M}$ and $\bar{\Gamma} \bar{K}$ are the [11 $\overline{2} 0]$ and [ $10 \overline{1} 0]$ directions, respectively.)

\footnotetext{
${ }^{*}$ Research sponsored by the U. S. Air Force Office of Scientific Research (AFSC) under Grant No. AFOSR 71-1973.

${ }^{1}$ R. E. Allen, G. P. Alldredge, and F. W. de Wette,
}

Phys. Rev. B $\underline{4}, 1661$ (1971).

${ }^{2}$ R. E. Allen and F. W. de Wette, Phys. Rev. 179,873 (1969). 